\title{
ESSAIS DE REMPLACEMENT \\ DU MILIEU D'ÉPREUVE GLYcosé de SABOURAUd \\ PAR LA GÉLOSE CZAPEK-DOX
}

Par Risto PÄTIÃLÃ

En étudiant des cultures de Trichophyton sulphureum, isolées par moi en Finlande, je me suis servi, sur le conseil de M. Langeron, de divers milieux, entre autres du Czapek-Dox gélosé.

J'ai alors remarqué que, sur ce milieu, les vrilles de ce dermatophyte étaient très nettes et bien développées, quoique petites, tandis que sur milieux naturels, tels que les grains d'orge ou le froment, on n'observait pas de vrilles, comme on aurait pourtant pu s'y attendre, d'après Langeron et Milochevitch (1930). Cette constatation m'a amené à étudier le développement d'autres dermatophytes sur le Czapek-Dox gélosé.

Comme termes de comparaison, j'ai pris le milieu d'épreuve glycosé de Sabouraud, mais modifié par Langeron, avec seulement 2 p. 100 de glycose.

Le milieu de Czapek a été préparé suivant la formule de Dox (1910):

\begin{tabular}{|c|c|}
\hline & $1.000 \mathrm{~cm}^{3}$ \\
\hline Nitrate de sodium & $3 \mathrm{gr}$. \\
\hline Phosphate bipotassique .... & $1 \mathrm{gr}$. \\
\hline Sulfate de magnésium ... & 0 gr. 5 \\
\hline Chlorure de potassium ........ & $0 \mathrm{gr} .5$ \\
\hline Sulfate de protoxyde de fer & 0 gr. 01 \\
\hline Sucrose (saccharose, sucre ordinaire). & 30 gr. \\
\hline Gélose & 15 \\
\hline
\end{tabular}

J'ai préparé aussi une autre formule de milieu de Czapek-Dox, en remplaçant les $30 \mathrm{gr}$. de sucrose par $20 \mathrm{gr}$. de glycose. Je désignerai ce milieu sous le nom de Czapek glycosé.

Mon but était de rechercher si d'autres milieux pouvaient fournir, pour les divers genres et espèces de dermatophytes, des images

Ann. de Parasitologie, T. XXII, Nos $3-4,1947$, p. 237-241. 
aussi typiques que celles données par le milieu d'épreuve glycosé de Sabouraud.

J'ai effectué ces recherches sur les espèces suivantes :

\begin{tabular}{||c|c|c|c||}
\hline Sabouraudites & Ctenomyces & Trichophyton & Epidermophyton \\
\cline { 5 - 6 } gudouini & $\begin{array}{c}\text { asteroides } \\
\text { granulosus } \\
\text { gallinæ }\end{array}$ & $\begin{array}{c}\text { crateriforme } \\
\text { sulphureum } \\
\text { lacticolor } \\
\text { pe:sicolor } \\
\text { interdigitalis } \\
\text { radians }\end{array}$ & $\begin{array}{c}\text { rubrum } \\
\text { violaceum } \\
\text { ferrugineum } \\
\text { album }\end{array}$ \\
\hline
\end{tabular}

Je n'ai pas cherché à savoir d'où provient la différence de croissance sur les divers milieux. Je ne me suis préoccupé que de l'aspect des colonies et des différences morphologiques microscopiques, ainsi que de l'apparition éventuelle du pléomorphisme.

L'examen macroscopique des colonies a été effectué au bout de $4,7,14,21,28$ et 50 jours; l'examen microscopique, après une, deux et trois semaines. J'ai effectué en outre des cultures sur lames gélosées, d'après Rivalier et Seydel, en ensemençant trois lames de chaque espèce et en les examinant ou bout d'une, deux et trois semaines.

Au bout de quatre jours, les Sabouraudites et Ctenomyces avaient déjà formé des colonies très nettes sur la gélose glycosée à 2 p. 100 , tandis qu'on ne voyait encore que des filaments sur les deux milieux de Czapek, sucrosé et glycosé.

Au bout de sept jours, les colonies étaient bien visibles sur les trois milieux ; néanmoins, elles étaient plus petites sur les deux milieux du type Czapek. La différence de coloration n'était pas particulièrement notable.

\section{Sabouraudites}

1. S. audouini. - Après 14 jours, larges colonies luxuriantes sur gélose glycosée, tandis que sur les deux milieux du type Czapek, on ne voyait aucun développement superficiel, toute la croissance ayant eu lieu à l'intérieur de la gélose (croissance immergée).

2. S. gypseus. - Colonies couleur lilas à la surface du milieu de 
Sabouraud, avec tendance au pléomorphisme. Colonies grises sur les deux milieux de Czapek.

3. S. gallinæ. - Larges colonies très développées sur gélose glycosée. Sur Czapek, croissance superficielle très faible, croissance profonde remplissant le tube.

\section{Ctenomyces}

1. C. asteroides. - Sur gélose glycosée, colonies bien développées, en partie granuleuses, en partie duveteuses. Sur Czapek, colonies bien développées, duveteuses, grisâtres.

2. C. granulosus. - Colonies blanches et duveteuses sur gélose g!ycosée, immergées et granuleuses sur les deux formules de Czapek.

3. C. lacticolor. - Grandes colonies duveteuses sur gélose glycosée, immergées et granuleuses sur les deux formules de Czapek.

4. C. persicolor. - Grandes colonies, pigmentées normalement, sur gélose glycosée, immergées et incolores sur les deux formules de Czapek.

5. C. interdigitalis. - Sur gélose glycosée, larges colonies blanches ; sur les deux formules de Czapek, colonies à peine visibles et grises en surface, à bords blanes, immergées et granuleuses.

6. C. radians. - Sur gélose glycosée, larges colonies opaques, avec rayons sur les bords et filaments stériles au centre. Sur les deux formules de Czapek, grandes colonies immergées, transparentes, à surface saupoudrée de flocons neigeux.

\section{TRICHOPHYTON}

1. T. crateriforme. - Sur gélose glycosée, larges colonies blondes; sur les deux formules de Czapek, colonies en mince couche grisâtre.

2. T. sulphureum. - Sur gélose glycosée, colonies jaunes, cratériformes; sur les deux formules de Czapek, colonies farineuses, jaunâtres.

3. T. rubrum. - Grandes colonies, très pigmentées, sur gélose glycosée; sur les deux formules de Czapek, colonies immergées, peu pigmentées, à peine duveteuses à la surface.

4. $T$. violaceum. - Sur gélose glycosée, petites colonies incolores, bleuâtres par endroits ; sur les deux formules de Czapek, très petites colonies immergées, à surface rougeâtre.

5. T. ferrugineum. - Sur gélose glycosée, grandes colonies rouil- 
lées, rayonnées, un peu surélevées. Sur les deux formules de Czapek, colonies à peine visibles en surface, immergées, peu colorées, formant un disque jaune de $1 \mathrm{~cm}$. environ de diamètre, entouré d'une frange grise de $2 \mathrm{~mm}$.

6. T. album. - Sur gélose glycosée, colonies luisantes, grises, larges de $1 \mathrm{~cm}$., hautes de $5 \mathrm{~mm}$. Sur Czapek au sucrose, petites cólonies à peine surélevées $(1 \mathrm{~mm}$.) ; sur Czapek glycosé, colonies entièrement immergées.

\section{EPIDERMOPHYTON}

E. floccosum. - Sur gélose glycosée, larges colonies jaunâtres, un peu pléomorphisées (duvet blanc). Sur les deux formules de Czapek, couche mince d'un duvet ressemblant à la fourrure de lemming, plus dense au centre.

D'une manière générale, la croissance des dermatophytes, sur les deux formules de Czapek sucrosé et glycosé, était plus lente que sur milieu glycosé et l'aspect des colonies ne correspondait pas aux belles images typiques, décrites par Sabouraud. En outre, surtout sur Czapek glycosé, les colonies avaient une grande tendance à se développer en profondeur et non en surface (colonies immergées).

Examen microscopique. - Malgré les espoirs qu'avait fait naître l'examen microscopique des colonies de Trichophyton sulphureum, développé sur milieu de Czapek gélosé, ce milieu ne se montra pas capable de produire une image morphologique complète.

Sabouraudites. - Très peu de différences dans les résultats obtenus avec les deux catégories de milieux.

Ctenomyces. - Morphologie microscopique meilleure sur Czapek, parce que les aleuries, se développant en moins grand nombre, ne cachaient pas les autres détails.

Trichophyton, - La morphologie microscopique, sur les deux formules de Czapek, se rapproche un peu de celle que donnent les milieux naturels.

Epidermophyton. - Même résultat pour les trois milieux.

En général, le Czapek glycosé a donné beaucoup de chlamydospores, mais n'a pu développer une image morphologique typique.

Pléomorphisme. - Il est resté nul sur le Czapek sucrosé, tandis qu'il s'est montré aussi bien sur milieu glycosé peptoné que sur Czapek glycosé. Ceci confirme les conclusions de Langeron et Milochevitch en ce qui concerne l'action favorisante du glycose sur le développement du pléomorphisme chez les dermatophytes. 


\section{RÉSUMÉ}

11 résulte de ces observations que les milieux d'épreuve de Sabouraud restent la base de l'étude des dermatophytes. Le milieu de Czapek, sucrosé ou glycosé, ne peut fournir l'aspect macroscopique typique, nécessaire pour la détermination des dermatophytes. Le Czapek original (sucrosé) peut donner une morphologie microscopique suffisante, ce que ne peut faire le Czapek glycosé, qui est donc tout à fait impropre à l'étude des dermatophytes.

\section{BiBLIOGRAPHIE}

Dox (A.-W.). - The intracellular enzymes of Penicillium and Aspergillus with special reference to those of P. camemberti. Bull. U.S. Dept. Agric. Bur. animal industry, $\mathrm{n}^{\circ} 120,1910$.

Langeron (M.) et Milochevitch (S.). - Morphologie des dermatophytes sur milieux naturels et milieux à base de polysaccharides. Essai de classification, Ann, de paras., VIII, 1930, p. 465-508, pl. XVII-XXVI,

PäträLä (R.). - Sur un cas de trichophytie produit par le Trichophyton sulphureum en Finlande. Ann. de paras., XXI, 1946, p. 336-337.

Sabouraud (R.). - Les teignes, Paris, Masson et Cie, 1910.

Institut de Parasitologie de la Faculté de Médecine de Paris (Directeur : Prof. E. Brumpt), Section de Mycologie médicale (Chef de service: $D^{r} M$. Langeron). 